\title{
ENTROPY CONDITIONS AND THEIR NUMERICAL ANALOGUES FOR CONSERVATION LAWS
}

\author{
R. ANSORGE
}

Institute of Applied Mathematics, University of Hamburg

Bundesstr. 55, D-2000 Hamburg 13, Germany

1. Entropy conditions and identity of corresponding entropy solutions. Many problems in applications are mathematically modeled by systems of conservation laws (e.g. in gas dynamics: conservation of energy, of momentum, of mass).

As a test problem, let us restrict ourselves to only scalar dynamical problems with one space variable (e.g. as a model of certain one-dimensional flows):

$$
\begin{gathered}
u_{t}+\frac{\partial}{\partial x} q(u)=0, \quad x \in \mathbb{R}, t \geq 0, \\
u(x, 0)=u_{0}(x) .
\end{gathered}
$$

The $\operatorname{flux} q: \mathbb{R} \rightarrow \mathbb{R}$ is assumed to be strictly convex, smooth (i.e. $C^{1}$ ) and without loss of generality -

$$
\min _{u \in \mathbb{R}} q(u)=q(0)=0 .
$$

Solutions - if they exist - are constant along the characteristics which are straight lines:

$$
x-x_{0}=q^{\prime}\left(u_{0}\left(x_{0}\right)\right) \cdot t .
$$

But because characteristics can intersect, discontinuities can occur such that the differential equations must be formulated in a weak form.

Lax [7] and Oleĭnik [11] used the formulation

(2) $-\iint_{\Omega}\left[u \cdot \Phi_{t}+q(u) \cdot \Phi_{x}\right] d x d t-\int_{\mathbb{R}} u_{0}(x) \cdot \Phi(x, 0) d x=0, \quad \forall \Phi \in C_{0}^{1}(\Omega)$,

1991 Mathematics Subject Classification: 35D99, 35F20, 65M05, 65M10, 76N15.

The paper is in final form and no version of it will be published elsewhere. 
which arises from the differential equation (1) by formal multiplication with test functions $\Phi$ and integration by parts.

Here, $C_{0}^{1}(\Omega)$ is the set of $C^{1}$ functions in the upper $(x, t)$ half-plane $\Omega$ having compact support.

We assume that there are solutions of this problem in $L_{1}^{\text {loc }}$, called weak solutions of the original problem, provided that

$$
u_{0} \in B V(\mathbb{R}) \cap L_{\infty}(\mathbb{R}) \quad \text { or } \quad u_{0} \in C(\mathbb{R}) \cap L_{\infty}(\mathbb{R}) .
$$

It can easily be shown by simple examples that weak solutions are not necessarily unique, but let us assume that $u_{0}$ is such that the solutions $u(x, t)$, arising from this $u_{0}$, are piecewise smooth functions of $x$ for every fixed $t>0$ and that discontinuities — as far as they occur — form smooth curves in the half-plane $\Omega$. These curves are called shocks.

If, on a time level $t, x$ tends to a point of a shock from the left, $u$ tends to a value called $u_{l}$. If $u_{r}$ is the value from the right, and if

$$
[u]=u_{l}-u_{r}, \quad[q]=q\left(u_{l}\right)-q\left(u_{r}\right)
$$

are the jumps, it can be shown just by means of the divergence theorem that

$$
[u] \cdot s=[q] \quad \text { (Rankine-Hugoniot) }
$$

where $s=d x / d t$ is the velocity of the moving discontinuity.

Moreover, the validity of the Rankine-Hugoniot condition along the shocks, together with the validity of the conservation law in its differential form (1) outside the shocks, is even equivalent to the weak formulation (2) of the problem.

Because weak solutions are not necessarily unique, one is interested in applications like gas dynamics, traffic flow models etc., in a criterion that allows to pick out of the set of solutions the physically true solution.

Lax [8] recommended to complete the weakly formulated conservation laws by an additional condition of the following type:

Find the particular weak solution $u_{E}$ which additionally satisfies the following condition:

There is a strictly convex smooth functional $V(u)$ and a smooth functional $F(u)$ such that automatically

$$
V_{t}(u)+\frac{\partial}{\partial x} F(u)=0
$$

for every weak solution $u$ in regions where this solution is smooth, and that the inequality

$$
V_{t}\left(u_{E}\right)+\frac{\partial}{\partial x} F\left(u_{E}\right) \leq 0
$$

holds weakly along every shock curve of $u_{E}$.

Some remarks should be made concerning this definition of uniqueness condition: 
- Weakly means that the last inequality reads

(6)

$$
\begin{array}{r}
-\iint_{\Omega}\left[V(u) \Phi_{t}+F(u) \Phi_{x}\right] d x d t-\int_{\mathbb{R}} V\left(u_{0}(x)\right) \Phi(x, 0) d x \leq 0, \\
\forall \Phi \in C_{0}^{1}(\Omega), \Phi \geq 0 .
\end{array}
$$

- Because Lax was led to this definition by the $2^{\text {nd }}$ main theorem of thermodynamics if applied to gas flows, the last condition is called the entropy condition, $V$ is called the entropy functional, and $F$ is the entropy flux.

- Condition (4) models the fact that the physical entropy satisfies automatically a conservation law, too, provided that the conservation of mass, of energy, and of momentum is respected and that shocks do not occur in the area under consideration.

Condition (4) establishes a connection between $F$ and $V$, namely

$$
F(u)=\int_{0}^{u} V^{\prime}(\alpha) q^{\prime}(\alpha) d \alpha+\gamma \quad(\gamma=\text { const })
$$

An example is

$$
V(u)=\frac{1}{2} \cdot u^{2} \Rightarrow F(u)=u q(u)-\int_{0}^{u} q(\alpha) d \alpha+\gamma .
$$

- The same arguments which led to the Rankine-Hugoniot condition (3) show that (6) is equivalent to the validity of the original differential equation problem (1) in regions where $u$ is a smooth solution, together with the validity of

$$
[V] \cdot s \leq[F]
$$

hence with

$$
\frac{[V][u]}{[q]} \leq[F]=\int_{u_{r}}^{u_{l}} V^{\prime}(\alpha) q^{\prime}(\alpha) d \alpha,
$$

and this — as can easily be shown — is equivalent to

$$
u_{r} \leq u_{l}
$$

independently of the pair $V, F$.

Because of the strict convexity of $q,(10)$ together with (3) implies in case of $u_{r}<u_{l}$ the relation

$$
\left(u_{l}-u_{r}\right) \cdot s=q\left(u_{l}\right)-q\left(u_{r}\right)=q^{\prime}(\widehat{u})\left(u_{l}-u_{r}\right),
$$

i.e.

$$
q^{\prime}\left(u_{r}\right)<s<q^{\prime}\left(u_{l}\right) .
$$

In 1973, Lax [9] showed that there is at most one weak solution that satisfies (2) and (11) 
Hence, the solution $u_{E}$, which we will call the Lax entropy solution (or shorter: Lax solution) is unique in the scalar case.

Taking the ascent $\left[q^{\prime}\left(u_{0}\left(x_{0}\right)\right)\right]^{-1}$ of the characteristics into account, (11) means geometrically that the entropy solution is characterized by the fact that the characteristic lines run into the shock for increasing time instead of leaving it.

Remark. It is (11) that was generalized by Lax in order to establish an entropy condition for arbitrary hyperbolic conservation law systems.

The problem of uniqueness was also treated by Olelnik [11]. She added instead of (4), (5) — the following condition:

Find the particular weak solution which additionally satisfies

$$
\frac{u(x+a, t)-u(x, t)}{a} \leq \frac{E}{t}, \quad \forall a>0, \forall t>0
$$

with a certain constant $E>0$.

Obviously, (12) implies (10) such that an Olěnik solution is at the same time a Lax solution and - hence - unique.

It should be mentioned that Oleĭnik had given a uniqueness proof, too, based upon (12), independently of Lax.

Instead of an entropy condition (6) with a smooth and strictly convex functional $V(u)$ (and a corresponding functional $F$ from $(7)$ ), one can find in the literature also functionals which are only continuous and piecewise linear but depend on an additional parameter $c$, e.g.

$$
V=|u-c|
$$

or

$$
V= \begin{cases}u-c, & u>c \\ 0, & u \leq c\end{cases}
$$

The validity of (6) is now not required to be satisfied for a strictly convex functional but only for one of these convex functionals; this is compensated by the condition that (6) is required to hold for all $c \in \mathbb{R}$.

Again, it can be shown that each of the conditions is then equivalent to the jump relation (10) provided that $F$ is chosen by (7), and upon the agreement that $V^{\prime}=V_{u}$ must be understood piecewise, and where $\gamma$ is allowed to depend on $c$.

Thus, if a weak solution of (1) additionally satisfies (13) or (14), this solution is the Lax-Oleinik solution and satisfies each of the entropy conditions mentioned before, for every $\gamma($ or $\gamma(c))$ in $(7)$.

We call (13) and (14) Kruzhkov entropy conditions.

The reason is that Kruzhkov [6] introduced in 1969 another transition from the original formulation of problem (1) to a weak formulation: 
A function $u \in L_{1}^{\mathrm{loc}}(\Omega)$ is called a weak solution of (1) if

$$
\begin{array}{r}
\iint_{\Omega}\left\{|u(x, t)-c| \Phi_{t}+\Phi_{x} \cdot \operatorname{sgn}(u(x, t)-c) \cdot[q(u(x, t))-q(c)]\right\} d x d t \geq 0, \\
\forall c \in \mathbb{R}, \forall \Phi \in C_{0}^{1} \text { with } \Phi \geq 0 \text { and with } \Phi(x, 0)=0,
\end{array}
$$

and if there is a set $\Upsilon \subset[0, T]$ of measure zero such that $u(x, t)$ is defined on $\Omega$ a.e. and for $t \in[0, T] \backslash \Upsilon$, and with

$$
\lim _{\substack{t \rightarrow 0 \\ t \in[0, T] \backslash \Upsilon}} \int_{-R}^{R}\left|u(x, t)-u_{0}(x)\right| d x=0, \quad \forall R \in \mathbb{R}^{+} .
$$

It follows easily that the Lax-Oleinik solution satisfies (15): Indeed, it is an element of $L_{1}^{\text {loc }}$, and if $V$ is chosen by $(13)$, if $\gamma(c)$ is chosen as

$$
\gamma(c)=q(c) \cdot \operatorname{sgn} c,
$$

and if $\Phi(x, 0)=0$ is taken into account, (15) is nothing but the entropy condition (6).

It is not as easy to show that the Lax-Oleinik solution satisfies (16), too.

But it can be shown that a numerical finite difference procedure which is consistent with the given problem (1) and which satisfies the assumptions of the Lax-Wendroff theorem, generates numerical approximations which converge for decreasing step sizes to a weak solution of $(1)$ in the $L_{1}^{\text {loc }}$-topology. Moreover, if it can be ensured that this limit is the Lax-Olelnik solution and that it satisfies relation (16) at the same time, the Lax-Oleınik solution is a Kruzhkov solution, too.

But because Kruzhkov showed that his weak solution is unique, we end up with

$$
u_{E_{\text {Oleŭnik }}}=u_{E_{\text {Lax }}}=u_{E_{\text {Kruzhkov }}}
$$

provided that there is such a numerical method.

We are going to show that such procedures exist, and it is at this point that we need our assumption

$$
u_{0} \in B V(\mathbb{R}) \cap L_{\infty}(\mathbb{R}) \quad \text { or } \quad u_{0} \in C(\mathbb{R}) \cap L_{\infty}(\mathbb{R}) .
$$

2. Discretized entropy conditions. We consider explicit finite difference methods of conservation form, i.e. methods which imitate the conservation form of the original problem (1):

$$
\frac{u_{i}^{\nu+1}-u_{i}^{\nu}}{\Delta t}+\frac{g_{i+1 / 2}^{\nu}-g_{i-1 / 2}^{\nu}}{\Delta x}=0,
$$

where

$$
g_{i+1 / 2}^{\nu}=g\left(u_{i+k}^{\nu}, u_{i+k-1}^{\nu}, \ldots, u_{i-p}^{\nu}\right)
$$

depends on $p+k+1$ variables. 
Here $u_{i}^{\nu}$ is expected to be an approximation of $u\left(x_{i}, t_{\nu}\right), x_{i}=i \cdot \Delta x, t_{\nu}=\nu \cdot \Delta t$, and the method will start from the discretized initial values

$$
u_{i}^{0}=\frac{1}{\Delta x} \int_{x_{i-1 / 2}}^{x_{i+1 / 2}} u_{0}(\xi) d \xi .
$$

The method is called a $(p+k+2)$-point method; e.g. for $p=0, k=1$ (three point method):

$$
u_{i}^{\nu+1}=u_{i}^{\nu}-\lambda \cdot\left\{g\left(u_{i+1}^{\nu}, u_{i}^{\nu}\right)-g\left(u_{i}^{\nu}, u_{i-1}^{\nu}\right)\right\}
$$

with $\lambda=\Delta t / \Delta x=$ const.

The method is called consistent with problem (1) if

$$
g(u, u, \ldots, u)=q(u), \quad \forall u \in \mathbb{R} .
$$

$g$ is called the numerical flux and is assumed to satisfy a Lipschitz condition.

Just for theoretical purposes, we extend the discrete approximate function to a $L_{1}^{\text {loc }}$-step function by

$$
u_{n}(x, t)=u_{i}^{\nu} \quad \text { for }\left\{\begin{array}{l}
x_{i-1 / 2} \leq x<x_{i+1 / 2} \\
t_{\nu} \leq t<t_{\nu+1}
\end{array}\right.
$$

where $\Delta x=O\left(\frac{1}{n}\right) ; \nu=0,1, \ldots, i=0, \pm 1, \pm 2 \ldots$

Already these assumptions ensure that limits $u$ of $L_{1}^{\text {loc }}$-convergent sequences $\left\{u_{n}\right\}$ of approximate solutions are weak solutions of (1) (Lax-Wendroff).

Whether or not the sequence $\left\{u_{n}\right\}$ is compact in $L_{1}^{\text {loc }}$, depends on $g$.

Examples are

$$
g_{i+1 / 2}=-\frac{1}{\lambda} \frac{u_{i+1}-u_{i}}{2}+\frac{q\left(u_{i+1}\right)+q\left(u_{i}\right)}{2}
$$

(Lax-Friedrich; also used by Oleŭnik for her uniqueness proof [11]) or

$$
g_{i+1 / 2}=q_{+}\left(u_{i+1}\right)+q_{-}\left(u_{i}\right)
$$

(Engquist-Osher [3]) with

$$
q_{-}(u)=\left\{\begin{array}{ll}
0, & u \geq 0, \\
q(u), & u<0,
\end{array} \quad q_{+}(u)= \begin{cases}q(u), & u \geq 0, \\
0, & u<0 .\end{cases}\right.
$$

Here, the flux $q$ is split in two parts according to the sign of the ascent of the characteristic lines; this is an old idea due to Courant, Isaacson, Rees [2]. Hence, the Engquist-Osher method is at the same time an example of the so-called flux splitting methods.

Weak solutions of (1) show the property of monotonicity, namely

$$
u_{0} \text { monotone } \Rightarrow u(\cdot, t) \text { monotone for every fixed } t>0 .
$$

Hence, also the numerical method should show this behavior, i.e.

$u_{i}^{0}$ monotone with respect to $i \Rightarrow u_{i}^{n}$ monotone with respect to $i$

$$
\text { for every fixed } n>0 \text {. }
$$


Such methods, in particular, avoid oscillations near shocks and are called monotonicity preserving.

Rem a r k. E.g., this is not respected by the Lax-Wendroff scheme [10], where

$$
g_{i+1 / 2}=q\left(u_{i+1}\right)+q\left(u_{i}\right) / 2-\lambda / 2 a_{i+1 / 2}\left[q\left(u_{i+1}\right)-q\left(u_{i}\right)\right]
$$

with

$$
a_{i+1 / 2}= \begin{cases}\left(q\left(u_{i+1}\right)-q\left(u_{i}\right)\right) /\left(u_{i+1}-u_{i}\right), & u_{i} \neq u_{i+1}, \\ q^{\prime}\left(u_{i}\right), & u_{i}=u_{i+1}\end{cases}
$$

Moreover, any weak solution of (1) satisfies

$$
\left\|u\left(\cdot, t_{2}\right)\right\|_{L_{1}} \leq\left\|u\left(\cdot, t_{1}\right)\right\|_{L_{1}} \quad \text { for } t_{2} \geq t_{1}
$$

in particular,

$$
\|u(\cdot, t)\|_{L_{1}} \leq\left\|u_{0}\right\|_{L_{1}}, \quad \forall t \geq 0 .
$$

Entropy solutions even show the property of $L_{1}$-contraction, i.e.

$$
\left\|u_{E}\left(\cdot, t_{2}\right)-v_{E}\left(\cdot, t_{2}\right)\right\|_{L_{1}} \leq\left\|u_{E}\left(\cdot, t_{1}\right)-v_{E}\left(\cdot, t_{1}\right)\right\|_{L_{1}} \quad \text { for } t_{2} \geq t_{1}
$$

if $u_{E}, v_{E}$ belong to $u_{0}, v_{0}$, respectively.

Thus, also this property should be imitated by the numerical method:

$$
\left\|u^{\nu+1}-v^{\nu+1}\right\|_{L_{1}} \leq\left\|u^{\nu}-v^{\nu}\right\|_{L_{1}}
$$

Another behavior of a weak solution is the fact that this solution is variation diminishing in time. Numerical procedures which imitate this property are called TVD schemes (Total Variation Diminishing).

It can easily be shown by means of the Lax-Wendroff theorem that TVD schemes of conservation form are convergent to a weak solution and, moreover, they are monotonicity preserving. Here, we assume that the Courant-FriedrichsLewy condition (CFL condition; [1])

$$
\lambda \leq \frac{1}{\left|q^{\prime}\right|_{\infty}^{*}}
$$

is satisfied, where

$$
\left|q^{\prime}\right|_{\infty}^{*}:=\max \left\{\left|q^{\prime}(u)\right|:|u| \leq\left\|u_{0}\right\|_{L_{\infty}}\right\} .
$$

Sufficient for TVD is the $L_{1}$-contractivity of the method.

Nevertheless, all these properties of a finite difference method are not yet sufficient to ensure that the limit of the approximate solutions is not some weak solution but the Lax-Oleınik entropy solution of problem (1).

But, fortunately, there is another characteristic property of this solution, namely its monotonicity with respect to the initial function:

(30) $\quad u_{0}(x) \geq v_{0}(x), \quad \forall x \in \mathbb{R}, \quad$ implies

$$
u(x, t) \geq v(x, t), \quad \forall x \in \mathbb{R}, \forall t>0 .
$$


The numerical method is called monotone if the analogous property

$$
u_{i}^{\nu} \geq v_{i}^{\nu}, \quad \forall i, \quad \text { implies } \quad u_{i}^{\nu+1} \geq v_{i}^{\nu+1}, \quad \forall i
$$

is satisfied. Then really the following theorem holds (Harten, Hyman, Lax [5]):

TheOREM. Method monotone $\Rightarrow u_{n} \rightarrow u_{E_{\text {Lax-Oleinik }}}$.

The disadvantage of monotone methods is their order which cannot exceed 1.

By the way, linear monotonicity preserving methods have to be monotone.

So, e.g., the Engquist-Osher scheme is monotone (also TVD etc.) but, hence, only of first order. The Lax-Wendroff scheme, on the other hand, is of order 2 , thus not a monotone method.

Therefore, particularly for higher order methods, we need another discretized version of an entropy characterizing property.

In order to construct such a discrete analogue, we start directly from the Lax-Oleunik entropy condition (5) of the original problem:

By a slight modification of the proof of the Lax-Wendroff theorem, it can easily be shown that the limit of the approximate solution sequence $\left\{u_{n}\right\}$ - if it exists - is the Lax-Oleinnik solution provided that the $u_{n}(n=1,2, \ldots)$ satisfy a discretized version of (5), namely

$$
\frac{V_{i}^{\nu+1}-V_{i}^{\nu}}{\Delta t}+\frac{G_{i+1 / 2}^{\nu}-G_{i-1 / 2}^{\nu}}{\Delta x} \leq 0
$$

with one of the entropy functionals $V$ occurring in (4) or taken from (13), (14), and with a suitable Lipschitz continuous numerical entropy flux G. Here,

$$
V_{i}^{\nu}=V\left(u_{i}^{\nu}\right), \quad G_{i+1 / 2}^{\nu}=G\left(u_{i+k}^{\nu}, u_{i+k-1}^{\nu}, \ldots, u_{i-p}^{\nu}\right) .
$$

The connection with (5) is required to be realized by another consistency condition, namely

$$
G(u, \ldots, u)=F(u)
$$

with $F$ from $(7)$.

But though this modification of the Lax-Wendroff theorem is often referred to in the literature, this concept is seldom concretely realized. Instead, alternative arguments are used or heuristic arguments or experimental experiences are often quoted if higher order methods are concerned.

Strack [13] really used (8) together with a numerical entropy flux obtained from a certain entropy flux splitting for three point schemes, namely

$$
G(\alpha, \beta)=F_{+}(\alpha)+F_{-}(\beta)
$$

with

$$
F_{+}(\alpha)=\left\{\begin{array}{ll}
F(\alpha), & \alpha \geq 0, \\
0, & \alpha<0 .
\end{array} \quad F_{-}(\beta)= \begin{cases}0, & \beta \geq 0 \\
F(\beta), & \beta<0\end{cases}\right.
$$

and he could show, at least for the Engquist-Osher scheme (23), that (32) holds locally, i.e. if a suitable step size control is observed.

Obviously, the splitting (34) satisfies the consistency condition (33). 
On the other hand, we already know by the monotonicity argument that the Engquist-Osher scheme approximates the Lax-Oleinnik entropy solution. Hence, Strack's result seems to be useless.

Nevertheless, let us show a particular way to prove the corresponding (and well known) global result using other arguments than Strack's, and this just as an example for a more complicated application of these considerations to higher order methods, such that also in such cases the convergence to the entropy solution can be completely shown.

Instead of $V(u)=\frac{1}{2} u^{2}$, used by Strack, we use the equivalent Kruzhkov type entropy functional (14), i.e.

$$
V(u ; c)= \begin{cases}u-c, & u>c \\ 0, & u \leq c,\end{cases}
$$

hence, by (7),

$$
F(u ; c)= \begin{cases}q(u)-q(c), & u>c \geq 0 \\ 0, & u \leq c, c \geq 0 \\ q(u), & u>c, c<0 \\ q(c), & u \leq c<0\end{cases}
$$

(if we choose $\gamma=0$ ).

Remark. It should be mentioned that Harten, Hyman, Lax showed their theorem mentioned above by proving that a monotone scheme satisfies (32), where they also used the same Kruzhkov entropy functional $V$, and with

$$
G_{i+1 / 2}=\sum_{j=i-k+1}^{i+k} V^{\prime}\left(u_{j}\right)\left(u_{j}-c\right) \int_{0}^{1} g_{u_{j}}\left(\widetilde{u}_{i-k+1}(\Theta), \ldots, \widetilde{u}_{i+k}(\Theta)\right) d \Theta
$$

where $\widetilde{u}(\Theta):=c+\Theta(u-c), 0 \leq \Theta \leq 1$.

But because we intend to consider also non-monotone methods of higher order, we ask for another realization of the numerical entropy flux.

If — as another example — Strack's numerical entropy flux splitting (34) is applied to (35), this leads to

$$
G(\alpha, \beta ; c)= \begin{cases}q(\alpha)-q(c), & \alpha>c>0, \\ 0, & \alpha \leq c, c>0, \\ 0, & \alpha \leq 0, \beta>0, \\ q(\beta), & \alpha \leq 0, c<\beta \leq 0, \\ q(\alpha), & \alpha>0, \beta>0, c \leq 0, \\ q(c), & \alpha \leq 0, \beta \leq c<0, \\ q(\alpha)+q(\beta), & \alpha>0, c<\beta \leq 0, \\ q(\alpha)+q(c), & \alpha>0, \beta \leq c \leq 0 .\end{cases}
$$

(35), (36) are still independent of the particular three point scheme under consideration. 
In order to check whether or not (32) is satisfied, a lot of cases must be taken into account for arbitrary fixed $i$, e.g. (we omit the superscript $\nu$ of $u_{i}^{\nu}$ ):

$$
\begin{array}{ll}
u_{i-1}, u_{i}, u_{i+1} \leq 0, & c \geq 0, \\
0<u_{i-1} \leq c, & u_{i} \leq 0, u_{i+1} \leq 0, \\
0<u_{i-1}, u_{i} \leq c, & u_{i+1} \leq 0, \\
\vdots & \\
c<u_{i} \leq 0, & u_{i-1}>0, u_{i+1}>0, \\
\vdots & \\
\text { etc. } \quad(36 \text { cases }) . &
\end{array}
$$

Also these necessary distinctions are independent of the three point scheme one is going to work with, but, evidently, the number of distinctions to be made increases with the number of points occurring in the multipoint scheme, also if $G$ is not constructed by flux splitting.

Let us now treat — as an example — the Engquist-Osher scheme.

Because of (19) and because this scheme is monotone, hence an $L_{1}$-contraction, we get

$$
\left|u_{i}^{\nu}\right| \leq\left\|u_{0}\right\|_{L_{\infty}}
$$

Thus, the Lipschitz continuity of $G(\alpha, \beta ; c)$ with respect to $\alpha, \beta$ must only be ensured for

$$
\alpha, \beta \in \mathbf{P}:=\left\{u:|u| \leq\left\|u_{0}\right\|_{L_{\infty}}\right\} .
$$

But because $q$ is in $C^{1}(\mathbf{P})$, this is obviously satisfied.

Of course, we cannot treat here all the 36 cases mentioned above, but this is no loss of probative force of our demonstration because this treatment is very similar in all these cases; it is really sufficient to study one of them in order to believe in the proposition for each of them:

Let

$$
u_{i+1}^{\nu} \leq c<u_{i}^{\nu} \leq 0, \quad u_{i-1}^{\nu} \leq 0 .
$$

In this case, the scheme gives

$$
\begin{aligned}
u_{i}^{\nu+1} & =u_{i}^{\nu}-\lambda\left\{q\left(u_{i+1}^{\nu}\right)-q\left(u_{i}^{\nu}\right)\right\} \\
& \leq u_{i}^{\nu}-\lambda\left\{q(c)-q\left(u_{i}^{\nu}\right)\right\}=u_{i}^{\nu}-\lambda\left(c-u_{i}^{\nu}\right) q^{\prime}\left(\widetilde{u}_{i}^{\nu}\right) .
\end{aligned}
$$

Hence,

$$
u_{i}^{\nu+1}-c \leq\left(u_{i}^{\nu}-c\right)\left[1+\lambda q^{\prime}\left(\widetilde{u}_{i}^{\nu}\right)\right]=\left(u_{i}^{\nu}-c\right)\left[1-\lambda\left|q^{\prime}\left(\widetilde{u}_{i}^{\nu}\right)\right|\right]
$$

But

$$
1-\lambda\left|q^{\prime}\left(\widetilde{u}_{i}^{\nu}\right)\right| \geq 1-\lambda\left|q^{\prime}\right|_{\infty}^{*} \geq 0
$$

(cf. (29), (37)), and $u_{i}^{\nu}-c>0$. 
Thus, the right hand side of (39), and therefore of (38), is non-negative. But the right hand side of (38) equals

$$
V_{i}^{\nu}+\lambda\left\{G_{i+1 / 2}^{\nu}-G_{i-1 / 2}^{\nu}\right\}
$$

indeed:

and (cf. (36))

$$
V_{i}^{\nu}=u_{i}^{\nu}-c
$$

$$
G_{i+1 / 2}^{\nu}=G\left(u_{i+1}^{\nu}, u_{i}^{\nu} ; c\right)=q(c), \quad G_{i-1 / 2}^{\nu}=G\left(u_{i}^{\nu}, u_{i-1}^{\nu} ; c\right)=q\left(u_{i}^{\nu}\right) .
$$

Therefore, (32) is satisfied independently of the sign of $u_{i}^{\nu+1}-c$.

All the other cases can be treated similarly so that (32) is really globally satisfied by the Engquist-Osher scheme for all $c \in \mathbb{R}$. Thus, this scheme satisfies (32) globally also if one of the other entropy functionals $V$ is used.

By the way, every entropy preserving numerical method whose solutions are compact in $L_{1}$ for increasing $n$, obviously converges to the Lax-Oleunik solution and satisfies - as already mentioned - (15), too. Provided that $u_{0} \in B V(\mathbb{R}) \cap$ $L_{\infty}(\mathbb{R})$, also (16) can be shown to be satisfied. This also holds by means of Lebesgue point arguments if $u_{0} \in C(\mathbb{R}) \cap L_{\infty}(\mathbb{R})$. Hence, the existence of methods that satisfy all the conditions we need (e.g. the Engquist-Osher scheme) finally now also establishes (17).

We are going to treat similarly the highly non-linear $2^{\text {nd }}$ order Harten 5-point scheme [4] he obtained by applying a non-oscillatory first order accurate method to an appropriately modified flux function.

This method is characterized by

$$
\begin{aligned}
g_{i+1 / 2} & =g\left(u_{i+2}, u_{i+1}, u_{i}, u_{i-1}\right) \\
& =\frac{1}{2}\left(q^{*}\left(u_{i+1}\right)+q^{*}\left(u_{i}\right)\right)-\frac{1}{2 \lambda} Q\left(\lambda a_{i+1 / 2}^{*}, \delta\right)\left(u_{i+1}-u_{i}\right) .
\end{aligned}
$$

Here,

$$
q_{i}^{*}=q\left(u_{i}\right)+\frac{1}{\lambda} h_{i}
$$

is a modified flux where $h_{i}=h\left(u_{i+1}, u_{i}, u_{i-1}\right)$ is a suitable function to be chosen (see below),

$$
a_{i+1 / 2}^{*}:=\frac{q_{i+1}^{*}-q_{i}^{*}}{u_{i+1}-u_{i}}, \quad Q(\alpha, \delta):= \begin{cases}|\alpha|, & |\alpha|>\delta, \\ \delta, & |\alpha| \leq \delta\end{cases}
$$

with $\delta \in(0,1)$ sufficiently small. This method is TVD if the modified CFL condition $\lambda\left|a_{i+1 / 2}^{*}\right| \leq 1$ is satisfied.

The function $h$ is chosen as follows:

$$
h_{i}:=s_{i} \cdot \max \left\{0, \min \left[\sigma_{i+1 / 2} \cdot\left|u_{i+1}-u_{i}\right|, s_{i} \cdot \sigma_{i-1 / 2}\left(u_{i+1}-u_{i}\right)\right]\right\},
$$

where

$$
s_{i}=\operatorname{sgn}\left(u_{i+1}-u_{i}\right), \quad \sigma_{i+1 / 2}=\frac{1}{2} Q\left(\lambda a_{i+1 / 2}, \delta\right)-\frac{1}{2}\left(\lambda a_{i+1 / 2}\right)^{2},
$$




$$
a_{i+1 / 2}=\frac{q\left(u_{i+1}\right)-q\left(u_{i}\right)}{u_{i+1}-u_{i}} .
$$

Having the Engquist-Osher example treated above in mind, Reuter recently [12] really proved the entropy preserving character of this $2^{\text {nd }}$ order Harten scheme:

He used the same Kruzhkov entropy functional $V$ we used for the EngquistOsher method with the corresponding entropy flux $F$ from (35). The only additional requirement is the restriction to only non-negative values of the parameter $c$ and therefore to non-negative initial functions $u_{0}$. Because Harten's method also guarantees $u_{i}^{\nu} \geq 0$ provided that $u_{0} \geq 0$ and that the step sizes are sufficiently small etc., and because $u_{0}$ represents a state variable as far as physical applications are concerned, this is no restriction from the point of view of these applications.

Reuter's numerical entropy flux is

$$
\begin{aligned}
G_{i+1 / 2} & =G\left(V_{i+2}, V_{i+1}, V_{i}, V_{i-1}\right) \\
& =\frac{1}{2} \cdot\left(F_{i+1}^{*}+F_{i}^{*}\right)-\frac{1}{2 \lambda} \cdot Q\left(\lambda A_{i+1 / 2}^{*}, \delta\right) \cdot\left(V_{i+1}-V_{i}\right)
\end{aligned}
$$

with

$$
\begin{gathered}
V_{i}=V\left(u_{i} ; c\right), \quad F_{i}^{*}=F^{*}\left(u_{i} ; c\right)=F\left(u_{i} ; c\right)+\frac{1}{\lambda} \cdot H_{i}, \\
H_{i}=H\left(V_{i+1}, V_{i}, V_{i-1}\right) \\
=S_{i} \cdot \max \left\{0, \min \left[\Sigma_{i+1 / 2} \cdot\left|V_{i+1}-V_{i}\right|, S_{i} \cdot \Sigma_{i-1 / 2} \cdot\left(V_{i}-V_{i-1}\right)\right]\right\},
\end{gathered}
$$

where

$$
\begin{gathered}
\Sigma_{i+1 / 2}=\frac{1}{2} \cdot Q\left(\lambda A_{i+\frac{1}{2}}, \delta\right)-\frac{1}{2} \cdot\left(\lambda A_{i+1 / 2}\right)^{2}, \quad S_{i}=\operatorname{sgn}\left(V_{i+1}-V_{i}\right) \\
A_{i+1 / 2}=\frac{F_{i+1}-F_{i}}{V_{i+1}-V_{i}}, \quad A_{i+1 / 2}^{*}=\frac{F_{i+1}^{*}-F_{i}^{*}}{V_{i+1}-V_{i}}
\end{gathered}
$$

Hence, this construction of the numerical entropy flux consists in replacing $g$ by $G, u_{i}$ by $V_{i}, q_{i}$ by $F_{i}$ etc.

Again a lot of different cases, namely sign combinations, have to be considered. As a matter of fact, there are more than 100 cases, but again all these cases can be treated very simply and very similarly just by means of the mean value theorem.

And the result really is that Harten's higher order scheme leads to the entropy solution, and this now without any doubts.

Thus, the Kruzhkov entropy functionals, though they are not strictly convex, are (in the scalar situation) of particular power if numerical analogs of the entropy condition, i.e. corresponding effective numerical entropy fluxes, are seeked in order to prove consistency of non-monotone numerical procedures with respect to the entropy condition of the original problem. 


\section{References}

[1] R. Courant, K. O. Friedrichs und H. Lewy, Über die partiellen Differenzengleichungen der mathematischen Physik, Math. Ann. 100 (1928), 32-74.

[2] R. Courant, E. Isaacson and M. Rees, On the solution of nonlinear hyperbolic differential equations by finite differences, Comm. Pure. Appl. Math. 5 (1952), 243-255.

[3] B. Engquist and S. Osher, Stable and entropy satisfying approximations for transonic flow calculations, Math. Comp. 34 (1980), 45-75.

[4] A. Harten, High resolution schemes for hyperbolic conservation laws, J. Comput. Phys. 49 (1983), 357-393.

[5] A. Harten, J. M. Hy man and P. D. Lax, On finite-difference approximations and entropy conditions for shocks, Comm. Pure Appl. Math. 29 (1976), 297-322 (with appendix by Barbara Keyfitz).

[6] S. N. Kružkov, Generalized solutions of the Cauchy problem in the large for nonlinear equations of first order, Soviet Math. Dokl. 10 (1969), 785-788.

[7] P. D. Lax, Weak solutions of nonlinear hyperbolic equations and their numerical computation, Comm. Pure Appl. Math. 7 (1954), 159-193.

[8] —, Shock waves and entropy, in: Contributions to Nonlinear Functional Analysis, E. Zarantello (ed.), Academic Press, New York 1971, 603-634.

[9] - Hyperbolic systems of conservation laws and the mathematical theory of shock waves, SIAM Regional Conference Series in Applied Mathematics 11 (1972), 48 pp.

[10] P. D. Lax and B. Wendroff, Systems of conservation laws, Comm. Pure Appl. Math. 13 (1960), 217-237.

[11] O. Oleŭnik, Discontinuous solutions of nonlinear differential equations, Amer. Math. Soc. Transl. Ser. 226 (1957), 95-172.

[12] S. Reuter, Die diskrete Entropiebedingung bei der numerischen Lösung skalarer Erhaltungsgleichungen, Diploma Thesis, Hamburg 1991.

[13] K. G. Strack, Discrete entropy condition and stability for conservation laws, Reports Inst. für Geom. und Prakt. Math., RWTH Aachen 30 (1985). 